\title{
Rank-level Fusion of Multispectral Palmprints
}

\author{
Neha Mittal \\ J.P. Institute of \\ Engineering \\ \&Technology, \\ Meerut, U.P. \\ India
}

\author{
Madasu \\ Hanmandlu \\ I.I.T. Delhi, \\ New Delhi-110016, \\ India
}

\author{
Jyotsana Grover \\ I.I.T. Delhi, \\ New Delhi-110016, \\ India
}

\author{
Ritu Vijay \\ Banasthali University, \\ Banasthali, \\ Rajasthan, India
}

\begin{abstract}
This paper presents an approach for the personal authentication using rank-level fusion of multispectral palmprints, instead of using multiple biometric modalities and multiple matchers. The rank level fusion involving the non linear combination of hyperbolic tangent functions gives the best recognition rate for the Rank 1 obtained from two types of features, viz., sigmoid and fuzzy. The results of using rank level fusion on the publicly available multispectral palmprint database show the significant improvement in the recognition rate as compared to the individual spectral bands. Recognition rate of $99.4 \%$ from sigmoid features and that of $99.2 \%$ from fuzzy features based on Rank 1 is the outcome of the hyperbolic tangent nonlinearity.
\end{abstract}

\section{General Terms}

Palmprint authentication, ROI, feature extraction, matching, fusion,

\section{Keywords}

Rank level fusion, Multi spectral palmprint, Borda Count, hyperbolic tangent.

\section{INTRODUCTION}

The palmprint as a biometric modality is slowly but decisively gaining acceptance in the field of biometrics. As compared to other biometric modalities, it is bestowed with enormous information in the literature which boosts its discriminating power. We will discuss a few important contributions that are made on this modality.

David Zhang et al. [1] have developed an online multispectral palmprint system. To examine the recognition performance of various spectral bands, a large multispectral palmprint database is created. The Red channel achieves the best result, whereas the Blue and Green channels have comparable performance but are slightly inferior to the Near Infrared Channel (NIC). A score level fusion scheme is proposed to integrate the features from multispectral palmprint. Multispectral fusion accompanied with higher recognition accuracy and antispoofing capability is superior to a single spectrum. Dong Han et al. [2] have adopted image fusion of multispectral palm using wavelet transform. The fusion is found to perform well on the blurred input images. Zhenhua Guo et al. [4] have analyzed the effect of seven different illuminations, including white light on the palmprint recognition. It is observed from the experimental results that the white light is not the optimal illumination. As different features of palm have different intensity distributions, the individual illumination can enhance different features of palm.
Nandakumar et al. [9] have suggested a hybrid fusion scheme that utilizes the ranked identities of matchers and their corresponding matching scores by using the Bayesian approach to improve the performance of the score-level fusion. Ajay Kumar and Sumit Shekhar[10] have utilized non linear functions like tanh, exponential functions for the rank level fusion of multiple palmprint representations. In [12], Ho et al. describe three methods (Highest rank, Borda count, and logistic regression) to combine the ranks assigned by different matchers. Monwar et al. [11] have consolidated the ranks of individual matchers (face, ear, and signature) using the highest rank, Borda count, and logistic regression approaches.

In this paper, we will explore fuzzy and sigmoid feature from the multispectral palmprints for the purpose of the rank level fusion.

The organization of the paper is as follows: Section 2 presents the extraction of fuzzy and sigmoid features. Matching, ranklevel fusion and results of implementation are described in Sections 3 and 4 and finally conclusions are given in Section 5.

\section{FEATURE EXTRACTION}

The palmprints are bestowed with random textures comprising crisscross lines in addition to three principal lines. We are concerned with only the random texture in this work. For this we demarcate the Region of Interest (ROI) from a palm. As the texture varies over ROI, it is partitioned into windows of appropriate size to represent the local texture.

Two types of features, fuzzy and sigmoid, are envisaged here. Both feature types are aimed at representing the uncertainty by treating the intensities in a window as forming a fuzzy set so that it can be fitted with a membership function.

\subsection{Fuzzy Features}

The intensities in a window may or may not follow a distribution when the size of the window is small. In that case, one can choose an arbitrary membership function. The cumulative response which is the defuzzified value computed from the intensities and membership values in a window represents the texture. The steps involved in the extraction of fuzzy features are outlined here.

\section{An Algorithm for Feature Extraction}

1. ROI is portioned into a fixed number of non-overlapping windows.

2. The average intensity $I_{\text {avg }}$ in a window of size $w x w$ is computed using

$$
I_{\text {avg }}=\frac{1}{W x W} \sum_{i=1}^{W} \sum_{j=1}^{W} I(i, j)
$$


3. The maximum intensity $I_{\max }$ in the window is found.

4. The membership function value $\mu_{\mathrm{ij}}$ for every pixel in the widow is computed using

$$
\mu_{i j}=1-\left(\frac{\left|I(i, j)-I_{a v g}\right|}{I_{\max }}\right)
$$

5. The cumulative response for $l^{\text {th }}$ window is obtained from:

$$
F(l)=\frac{\sum_{i=1}^{W} \sum_{j=1}^{W} \mu_{i j} . I(i, j)}{\sum_{i=1}^{W} \sum_{j=1}^{W} \mu_{i j}}
$$

The above value is used as a feature and the number of these features is equal to the number of windows in a ROI.

\subsection{Sigmoid Features}

For the extraction of sigmoid features, Steps 1-4 of the above algorithm are the same. Hence are not repeated here. However, we replace the fuzzy feature in (5) by the sigmoid function in Step 5 of the above algorithm. Accordingly, we have

5. The sigmoid function for $l^{\text {th }}$ window is computed from:

$$
\operatorname{Sig}(l)=\sum_{i=1}^{W} \sum_{j=1}^{W} \frac{I_{a v g}}{1+\exp \left(-\mu_{i j}\right)}
$$

Here the sigmoid function is evaluated over the window.

\section{MATCHING AND RANK LEVEL FUSION}

In this we will use a simple classifier like Euclidean distance classifier for determining the ranks at the matching stage and put in our effort on the fusion of ranks using non linear functions.

\subsection{Euclidean Distance Classifier}

Given a set of training features and another set of test features corresponding to the training and testing samples, the Euclidean distance classifier is invoked as a measure of dissimilarity for the palmprint matching. The features that enter the matching stage include both sigmoid and fuzzy.

\subsection{Rank Level Fusion}

The purpose of an identification system is to ascertain the unknown identity from a set of users present in a database based on the input biometric traits. The classifier (matcher) aids this by comparing the input (feature) data of unknown user against all the users (their features) in the database producing a set of dissimilarity scores calculated using the Euclidean distance. Then the scores are sorted in the ascending order to form the ranking list of matched users, i.e. a user with the lowest score is awarded the rank 1 indicating the best matched user and the next best matched user is given rank 2 and so on. So the rank information indicates the relative ordering of the scores of the different users. The task of the rank level fusion is to identify the person by adopting such a rank level strategy that makes use of the best of the individual matchers resulting in better recognition accuracy than that of the individual classifier/matcher. The rank-level fusion is considered for consolidating the ranked identities of users received from the multiple classifiers.

As an alternative to multiple classifiers and/or multibiometrics, we have used multispectral palmprint database [6] and investigated several rank-level fusion approaches in this study. These approaches include: Borda count, weighted Borda count, maximum rank method and/or non-linear weighted ranks [10].

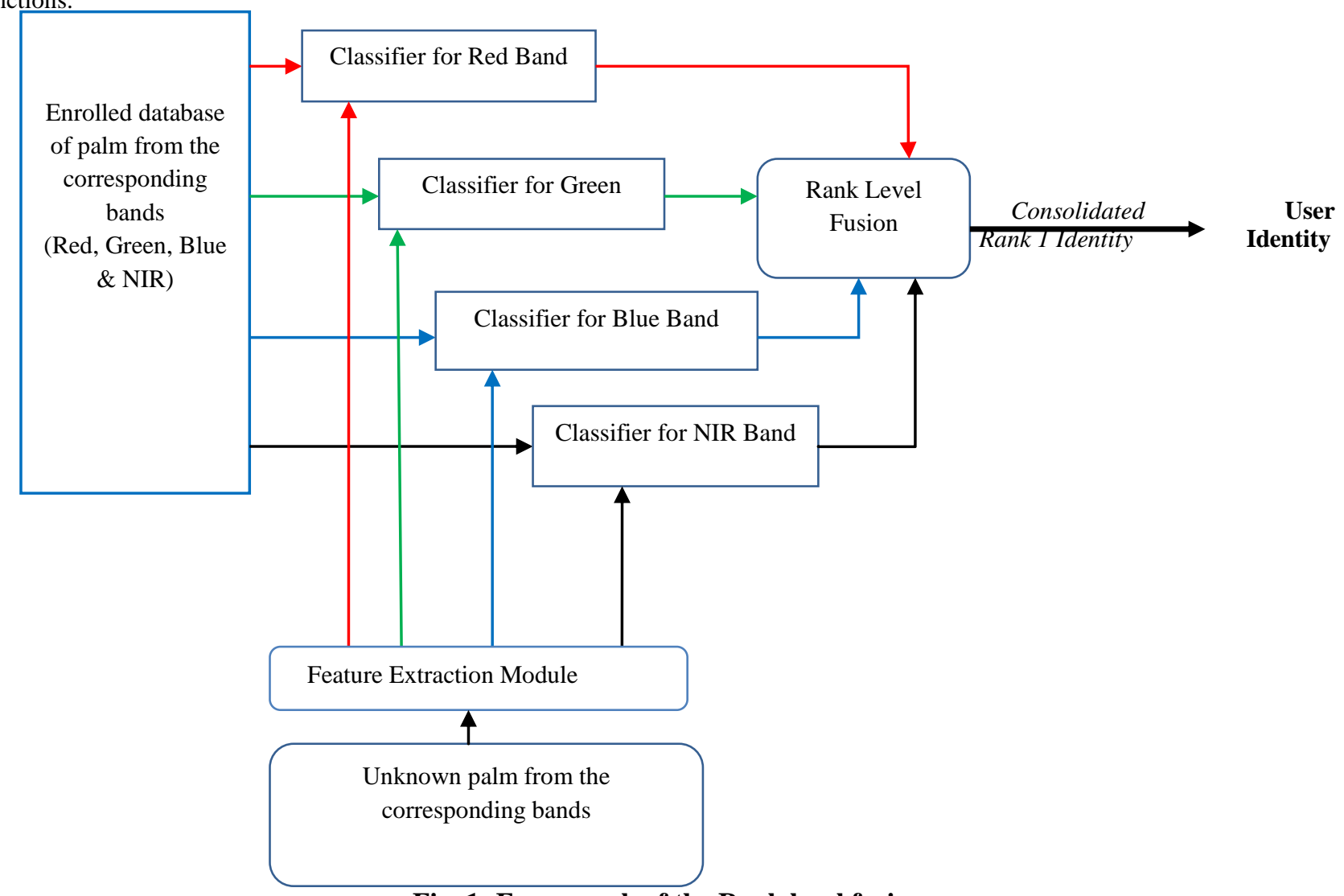

Fig. 1: Framework of the Rank level fusion 
Fig. 1 gives the block diagram of the rank level approach to find the user identity from the multispectral palmprints. This figure illustrates the classification of multispectral palmprints based on the ranked identities, which are used in the rank level fusion. The rank 1 identity is used to identify the unknown user. The rank level approaches investigated in this study are now briefed here.

\section{Borda Count}

In this the votes from the multispectral palmprint classifiers are added to identify an unknown user. The user with the highest votes is declared as the winner

\section{Weighted Borda Count}

In this method, weights are multiplied by the ranked output from the individual classifiers and then consolidated. These weights signify the importance of classifier. In this approach the weights are calculated according to the errors, see for example $\mathrm{w}_{\mathrm{i}}=1 / \mathrm{e}_{\mathrm{i}}$

\section{Maximum Rank Method}

In this method the highest rank is used to identify the unknown user.

\section{Nonlinear Weighted Ranks}

Let $\mathrm{w}_{\mathrm{i}}$ be the weight and $R_{\mathrm{ij}}$ be the rank assigned to the $\mathrm{j}^{\text {th }}$ user by $i^{\text {th }}$ spectral band. Weights are calculated using the logistic regression of the genuine scores as follows:

$$
\begin{gathered}
C_{j}=\sum_{i=1}^{N} \tanh \left(w_{i} * R_{i j}\right) \\
C_{j}=\sum_{i=1}^{N} a \sinh \left(w_{i} * R_{i j}\right) \\
C_{j}=\sum_{i=1}^{N} a \tanh \left(w_{i} * R_{i j}\right) \\
C_{j}=\sum_{i=1}^{N} \frac{1}{1+\exp \left(w_{i} * R_{i j}\right)} \\
C_{j}=\sum_{i=1}^{N} \log \left(1+w_{i} * R_{i j}\right)
\end{gathered}
$$

Where $j=1,2 \ldots M$ ( $M$ is the total number of users). $C_{j}$ are arranged in the ascending order. The Rank 1 is assigned to the user with the least value of $\mathrm{C}_{\mathrm{j}}$.

\section{RESULTS OF IMPLEMENTATION}

\subsection{Palmprint Database}

The database of multispectral palmprint images of 250 individuals is taken from the Biometric Research Centre (UGC/CRC) at The Hong Kong Polytechnic University. The database contains 195 males in the ages raging from 20 to 60 years. The acquisition of multispectral palmprint images is done on two separate sessions. The average time interval between the two occasions is 9 days. Six samples are collected from each subject from his/her left and right palms. The database contains 6000 images from 500 different palms for each band. Four images are taken in every shot from four bands (Red, Green, Blue, and NIR) in less than 1s. The resolution of the images is $352 \times 288(<100 \mathrm{dpi})$. Fig. 3 shows the multispectral palmprint ROI for RED band, Green band, Blue band and IR band respectively.

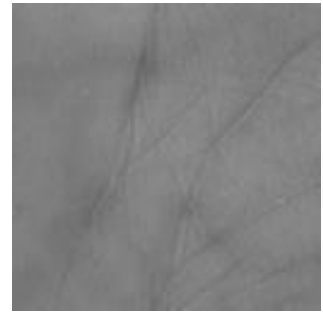

(a)

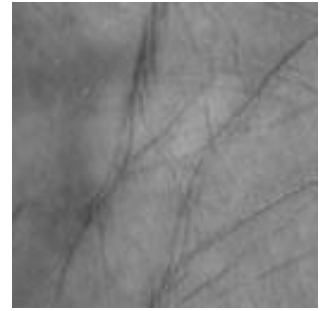

(c)

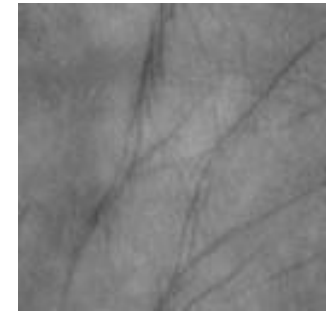

(b)

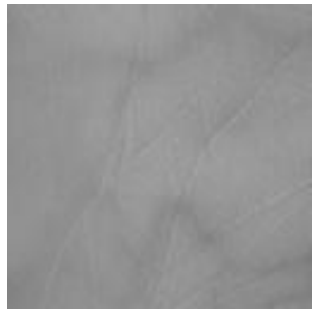

(d)
Fig. 2: Multispectral Palmprint ROI for Red Band (a): Green Band (b): Blue Band (c) and IR Band (d)

\subsection{Discussion of Results}

The rank level fusion is tested on multispectral palmprint database consisting of 500 subjects, 4 spectral bands (Red, Green, Blue and IR) for each subject and 6 samples for each band totaling 500x6x4=12,000 samples. In this 4 samples are taken as the training set and 2 as the testing set. The recognition rates of individual spectral bands from both types of features are given in Table 1 and those from different ranklevel-fusion schemes implemented on Ranks 1, 2 and 3 are given in Table 2. It is observed from the experimental results that the non linear approaches used for the rank level fusion are very effective in achieving good recognition rates. Hyperbolic tangent nonlinearity gives the best recognition rate on both types of features using Rank 1, while the maximum rank method fails to yield good recognition rate on Rank 1 but this method gives an improved rate of $99.46 \%$ on the lower ranks (i.e. Rank 3).

A comparison with the previous results is not possible as there is no work reported on rank level fusion of spectral images of palmprints. Ajay Kumar and Sumi Shekhar [10] have suggested that their non linear rank level combination achieves the best performance on palmprints. Exponential non linearity is found to give the best result on Rank 1. In our study this non linearity is not able to deliver the best performance whereas the hyperbolic tangent nonlinearity achieves the best performance.

TABLE 1. Recognition rate from individual spectral bands

\begin{tabular}{|l|l|l|}
\hline & Sigmoid & Fuzzy \\
\hline Red & 95.46 & 94.6 \\
\hline Green & 95.13 & 81.93 \\
\hline Blue & 95.53 & 98.93 \\
\hline NIR & 93.93 & 97.46 \\
\hline
\end{tabular}


Table 2. Recognition rate from various rank-level-combination schemes for sigmoid and fuzzy feature

\begin{tabular}{|c|l|l|l|l|l|l|}
\hline & \multicolumn{3}{|c|}{ Sigmoid feature } & \multicolumn{3}{c|}{ Fuzzy feature } \\
\cline { 2 - 7 } & Rank1 & Rank2 & Rank3 & Rank1 & Rank2 & Rank3 \\
\hline Borda Count & 95.53 & 96.46 & 97 & 89.8 & 91.8 & 92.866 \\
\hline Maximum Rank Method & 94.26 & 98.53 & 99.46 & 88 & 89.06 & 90.26 \\
\hline$\sum \tanh \left(w_{i}^{*} R_{i}\right)$ & $\underline{99.4}$ & 99.6 & 99.6 & 99.2 & 99.5 & 99.5 \\
\hline$\sum a \sinh \left(w_{i}^{*} R_{i}\right)$ & 98.06 & 99 & 99.2 & 96.6 & 98.66 & 99.33 \\
\hline$\sum a \tanh \left(w_{i} * R_{i}\right)$ & 99.4 & 99.53 & 99.53 & 98.06 & 98.6 & 98.6 \\
\hline$\sum \frac{1}{1+\exp \left(w_{i}^{*} R_{i}\right)}$ & 99.2 & 99.6 & 99.6 & 98.93 & 99.4 & 99.4 \\
\hline$\sum \log \left(1+w_{i} * R_{i}\right)$ & 98.8 & 99.2 & 99.4 & 98.4 & 99.3 & 99.4 \\
\hline
\end{tabular}

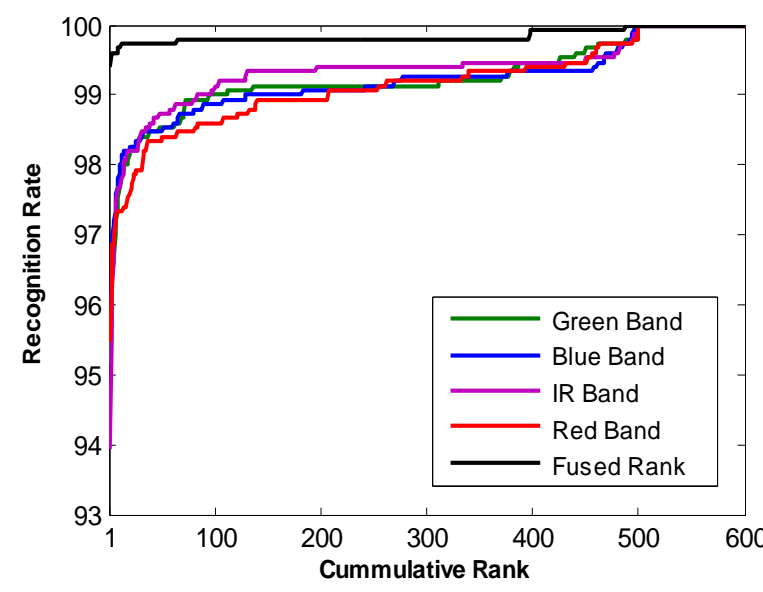

(a)

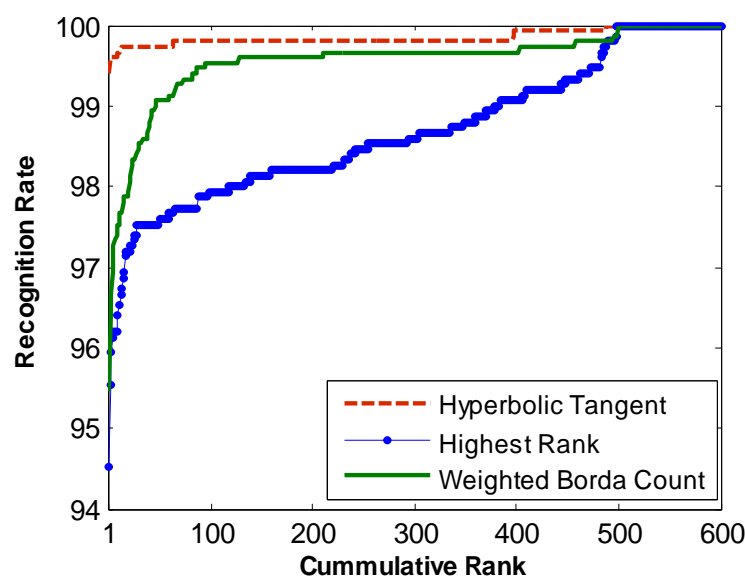

(b)

Fig. 3: The CMC for the performance from individual classifiers and rank-level combination using nonlinear (Hyperbolic Tangent) approach (a): CMC curve for the rank-level combination using highest rank method, Weighted Borda count and hyperbolic tangent non-linear approach from sigmoid feature (b)

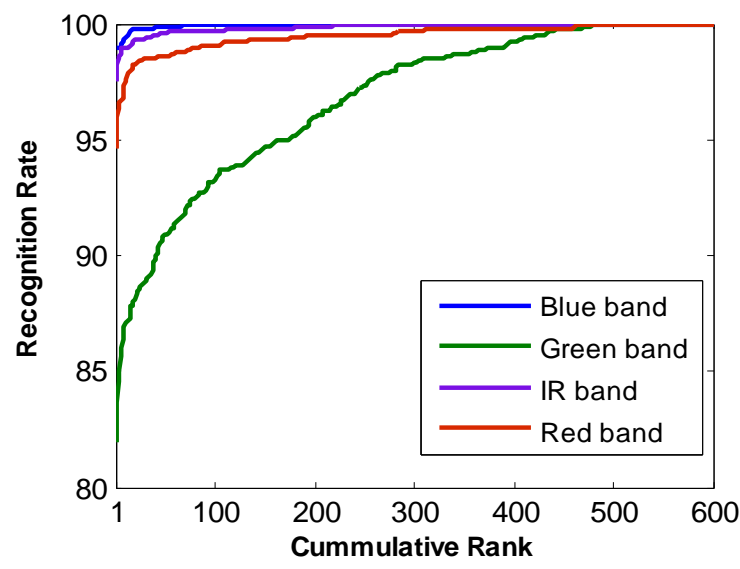

(a)

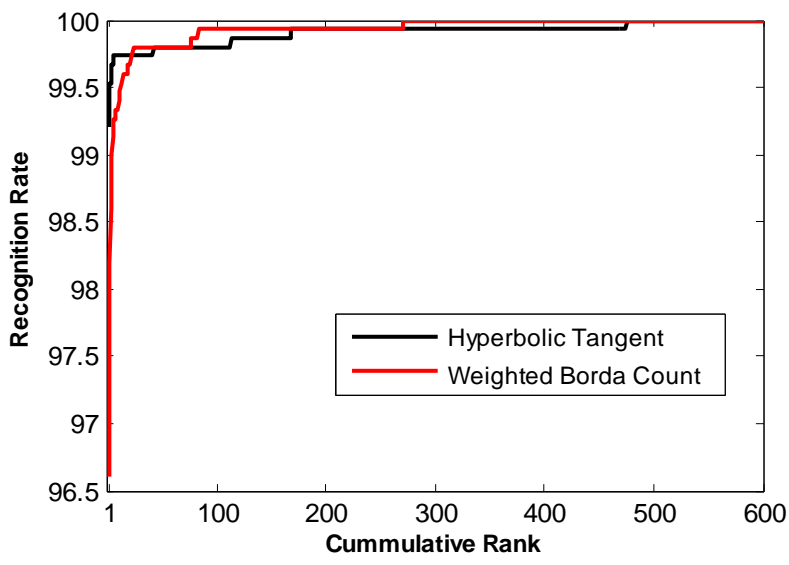

(b)

Fig. 4: performance from individual multispectral palmprint classifiers using CMC (a): CMC curve for the rank-level combination using weighted Borda count and hyperbolic tangent non-linear approach from fuzzy feature (b) 
Fig. 3 depicts the Cumulative Match Characteristics (CMC) of the individual classifiers and the rank level fusion using non linear (hyperbolic tangent) approach. As can be seen from the figure, the results are improved considerably at the lower ranks as compared to the individual spectral bands. Fig.4 shows the CMC for the performance from individual multispectral palmprint classifier in (a) and CMC curve for the rank-level combination using weighted Borda count and hyperbolic tangent nonlinear approach from fuzzy feature in (b).

\section{CONCLUSIONS}

There has been no effort on the rank level fusion on the multispectral palmprints. The spectral bands of palmprints are fused by the rank level fusion to achieve the significant improvement in the performance of the authentication system. The nonlinear combinations utilized in the rank level fusion are shown to outperform over the existing approaches: Borda count, weighted Borda count or maximum rank method. Out of the nonlinear functions implemented, the hyperbolic tangent function with Rank 1 is found to be superior to all others in terms of recognition rates.

\section{ACKNOWLEDGMENTS}

We thank Dr. Lei Zhang (Associate Professor), Poly Technique University, Hong Kong for providing multispectral palmprint database.

\section{REFERENCES}

[1] David Zhang, Zhenhua Guo, Guangming Lu, Lei Zhang, and Wangmeng Zuo, "An Online System of Multispectral Palmprint Verification", IEEE Transactions on Instrumentation and Measurement, vol. 59, no. 2, pp. 480-490, 2010.

[2] Dong Han, Zhenhua Guo, and David Zhang, "Multispectral Palmprint Recognition using waveletbased Image Fusion", International Conference on Signal Processing, pp. 2074-2077, 2008.

[3] David Zhang, Wai-kin Kong, Jane You and Michael Wong, "On-line palmprint identification", IEEE Transactions on Pattern Analysis and Machine Intelligence, vol. 25, no. 9, pp. 1041-1050, 2003.
[4] Zhenhua Guo, David Zhang, Lei Zhang, Wangmeng Zuo, Guangming Lu "Empirical Study of Light Source Selection for Palmprint Recognition", Pattern Recognition Letters (2010), doi: 10.1016/j. patrec.2010.09.026

[5] R. C. Gonzalez, R. E. Woods, Digital Image Processing, Addison Wesley publishers, 1993.

[6] H. K. Polytechnic University, "Multispectral Palmprint database", Biometric Research Center Website. http://www4.comp.polyu.edu.hk/ biometrics/.

[7] Madasu Hanmandlu, Ritu Vijay, Neha Mittal, "A Study of Some New Features for Palmprint Authentication", Proceedings of the World Congress on Engineering 2011, WCE 2011, 6-8 July, 2011, London, U.K., pp $1623-1628$

[8] M. Hanmandlu, H.M. Gupta, Neha Mittal, and S. Vasikarla, "An Authentication System Based on Palmprint", in Proc. ITNG, IEEE Computer Society, 2009, pp.399-404.

[9] K. Nandakumar, A. K. Jain, and A. Ross, "Fusion in multibiometric identification systems: What about the missing data?", Third Int. Conf. Biometrics (ICB), $743-$ 752,2009, Alghero, Italy.

[10] Ajay Kumar, and Sumit Shekhar, "Personal Identification Using Multibiometrics Rank-Level Fusion", IEEE Trans. on Systems, Man, and Cybernetics-Part C, Application and Review, 41 (5), 743-752, 2011.

[11] Md. Maruf Monwar, and Marina L. Gavrilova, 2009. Multimodal biometric system using rank-level fusion approach. IEEE Tran. on Systems, Man, and Cybernetics, Part B: Cybernetics - Special issue on cybernetics and cognitive informatics, 39 (4), $867-878$, 2009.

[12] T. K. Ho, J. J. Hull, and S. N. Srihari, 1994. Decision Combination in Multiple Classifier Systems. IEEE Trans. on Pattern Analysis and Machine Intelligence, 16 (1), 66$75,1994$. 